Supporting information

\title{
2D QSAR Consensus for High-Throughput Virtual Screening. Application to COX-2 inhibitors : Screening of the NCI database
}

Nicolas Baurin, Jean-Christophe Mozziconacci, Eric Arnoult, Philippe Chavatte, Christophe Marot, Luc Morin-Allory.

\section{QSAR methodology - supporting information}

BRANN : The three-layered architecture of our BRANN model is as following: an input layer of 49 neurons, a hidden layer of 12 tanh neurons with 1 bias each, and one output neuron, with bias, whose activation function is the identity. The value of the output neuron is the mean of a Gaussian distribution for the target, with the standard deviation of this Gaussian (the noise level) being a hyperparameter picked from an inverse Gamma prior distribution (specified by 0.05:0.5::4 according to Neal notation). Hyperparameters are also used that give the standard deviations of the Gaussian prior distributions for the network weights: one hyperparameter for each input-to-hidden units group of weights (ARD implementation, inverse gamma prior specification 0.2:0.5:0.5), one hyperparameter for the hidden unit biases (inverse gamma prior specification 0.05:0.5), one hyperparameter for the hidden-to-output weights (rescaled inverse gamma prior specification 0.05:0.5). The output unit bias is given a simple Gaussian prior with mean 0 and standard deviation 100. The prior distributions are sampled with Markov chains operations. These operations are Gibbs sampling for both the hyperparameters and the noise level, a heat bath update for the momentum variables, and a hybrid Monte Carlo update with a trajectory 1000 leapfrog steps long, a window of 10, and a stepsize adjustment factor of 0.4 . The model was trained during 2000 iterations, and one network out of 10 was finally retained for prediction once equilibrium was reached: that way, 171 networks, sampled from iterations 300 to 2000 , were used for prediction (committee prediction), i.e the mean of these 171 predictions is the final prediction we used.

GA-kNN : The GA is used to choose from the 49 initial P_VSA descriptors, the best combination for a good prediction. We used a multi-population GA (25 populations of 50 chromosomes each) with a scoring function $\mathrm{S}$ defined as: ${ }^{37}$

$$
\begin{aligned}
\mathrm{S} & =1-\left[(\mathrm{n}-1)^{*}\left(1-\mathrm{q}^{2}\right) /(\mathrm{n}-\mathrm{k}-1)\right] . \\
\mathrm{q}^{2} & =\frac{\sum_{p=1}^{n}\left(p I C_{50}^{\text {real }}{ }^{\text {rea }}-p I C_{50}{ }^{\text {predicted }}\right)^{2}}{\sum_{p=1}^{n}\left(p I C_{50}{ }^{\text {real }}-\overline{p I C_{50}{ }^{\text {real }}}\right)^{2}}
\end{aligned}
$$




$$
, \mathrm{n}=266
$$

The expression of S enables the GA to look for a combination of P_VSA descriptors with good predictive ability ( $\mathrm{q}^{2}$, defined by a Leave-One-Out procedure, closest to 1$)$, and a minimum number of neighbors (k) used. Simultaneously the GA is searching the best k value (from 1 to 5). The GA type for each population is «Steady-state » : 40\% of the 50 chromosomes are replaced each cycle (the cross-over probability is $100 \%$ [the probability to choose a chromosome from another population is $10 \%]$, the mutation probability is $2 \%$ ). We trained this GA for 1500 iterations and finally obtained a 19 P_VSA descriptors GA-kNN model with $\mathrm{k}=5$, used for final prediction.

LVQ : The initialization allocated 67 "Active" labelled vectors and 154 "Inactive" labelled vectors. We then used the oLVQ1 algorithm (16000 iterations, fast convergence), and then successive cycles of LVQ3 algorithm (1330 iterations, $\alpha(0)=0.03, \varepsilon=0.1, \omega=0.3)$ followed by LVQ2.1 algorithm (1330 iterations, $\alpha(0)=0.03, \omega=0,3)$ until stabilization of the classification performance on the 266 ligands training set. The finally retained LVQ model correctly classified $92 \%$ of the 179 "Inactive" COX-2 inhibitors (pIC50<7.5), and correctly classified $85 \%$ of the 87 "Active" COX-2 inhibitors (pIC50 $\geq 7,5$ ).

SOM : : The dimensions of the map is $20 * 20$, the topology is hexagonal, the learning rate function type is inverse-time: We first run a cycle with 1000 iterations (number of vectors considered in the neighborhood of the winning neuron $=15, \mathrm{C}=10$ and $\alpha(0)=0.05)$, followed by a second cycle with 10000 iterations (number of vectors considered in the neighborhood of the winning neuron $=3, \mathrm{C}=100$ and $\alpha(0)=0.02$ ). We made 20 different SOM maps and finally retained the map with the lowest weighted distance measured from the 266 reference vectors. Concerning the calibration step, when a vector was doubly labeled as both "Active" and "Inactive", we considered this vector as "Active" for the class prediction with the underlying idea that it is preferable not to miss a real "Active" compound. The final SOM map has 41 vectors labeled "Active", 50 vectors labeled "Inactive" and 309 non-labeled "vectors". 
Table I : Structures and COX-2 inhibition power of pyrrole derivatives.

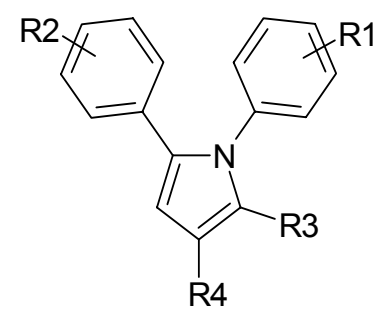

\begin{tabular}{|c|c|c|c|c|c|}
\hline Compound & R1 & $\mathbf{R 2}$ & $\mathbf{R 3}$ & $\mathbf{R 4}$ & pIC $_{\mathbf{5 0}}$ \\
\hline $\mathbf{1}$ & $4-\mathrm{F}$ & $4-\mathrm{SO} 2 \mathrm{Me}$ & $\mathrm{Me}$ & $\mathrm{H}$ & 7.22 \\
\hline $\mathbf{2}$ & $4-\mathrm{SO} 2 \mathrm{NH} 2$ & $4-\mathrm{F}$ & $\mathrm{H}$ & $\mathrm{H}$ & 7.85 \\
\hline $\mathbf{3}$ & $4-\mathrm{F}$ & $4-\mathrm{SO} 2 \mathrm{Me}$ & $\mathrm{Me}$ & $\mathrm{COCF} 3$ & 6.92 \\
\hline $\mathbf{4}$ & $4-\mathrm{F}$ & $4-\mathrm{SO} 2 \mathrm{Me}$ & $\mathrm{Me}$ & $\mathrm{COCH} 3$ & 5.79 \\
\hline $\mathbf{5}$ & $4-\mathrm{F}$ & $4-\mathrm{SO} 2 \mathrm{Me}$ & $\mathrm{Me}$ & $\mathrm{COPh}$ & 5.99 \\
\hline $\mathbf{6}$ & $4-\mathrm{F}$ & $4-\mathrm{SO} 2 \mathrm{Me}$ & $\mathrm{Me}$ & $\mathrm{CHO}$ & 5.49 \\
\hline $\mathbf{7}$ & $4-\mathrm{F}$ & $4-\mathrm{SO} 2 \mathrm{Me}$ & $\mathrm{Me}$ & $\mathrm{CN}$ & 6.12 \\
\hline $\mathbf{8}$ & $4-\mathrm{F}$ & $4-\mathrm{SO} 2 \mathrm{Me}$ & $\mathrm{Me}$ & $\mathrm{Br}$ & 7.7 \\
\hline $\mathbf{9}$ & $4-\mathrm{F}$ & $4-\mathrm{SO} 2 \mathrm{Me}$ & $\mathrm{Me}$ & $\mathrm{Cl}$ & 7.3 \\
\hline $\mathbf{1 0}$ & $4-\mathrm{F}$ & $4-\mathrm{SO} 2 \mathrm{Me}$ & $\mathrm{Me}$ & $\mathrm{CH} 2 \mathrm{~N}(\mathrm{CH} 3)_{2}$ & 4.0 \\
\hline $\mathbf{1 1}$ & $4-\mathrm{F}$ & $4-\mathrm{SO} 2 \mathrm{Me}$ & $\mathrm{Me}$ & $\mathrm{CH} 2 \mathrm{OCOCH}$ & 6.33 \\
\hline $\mathbf{1 2}$ & $4-\mathrm{F}$ & $4-\mathrm{SO} 2 \mathrm{Me}$ & $\mathrm{Me}$ & $\mathrm{CH} 2 \mathrm{OH}$ & 5.41 \\
\hline $\mathbf{1 3}$ & $4-\mathrm{SO} 2 \mathrm{CH} 3$ & $4-\mathrm{F}$ & $\mathrm{H}$ & $\mathrm{H}$ & 6.29 \\
\hline $\mathbf{1 4}$ & $4-\mathrm{F}$ & $4-\mathrm{SO} 2 \mathrm{Me}$ & $\mathrm{Me}$ & $\mathrm{CH}(\mathrm{OH}) \mathrm{CF} 3$ & 5.84 \\
\hline $\mathbf{1 5}$ & $4-\mathrm{F}$ & $4-\mathrm{SO} 2 \mathrm{Me}$ & $\mathrm{Me}$ & $\mathrm{CH} 2 \mathrm{CF} 3$ & 6.85 \\
\hline $\mathbf{1 6}$ & $4-\mathrm{F}$ & $4-\mathrm{SO} 2 \mathrm{NH} 2$ & $\mathrm{H}$ & $\mathrm{H}$ & 4.99 \\
\hline $\mathbf{1 7}$ & $\mathrm{H}$ & $4-\mathrm{SO} 2 \mathrm{Me}$ & $\mathrm{Me}$ & $\mathrm{H}$ & 7.22 \\
\hline $\mathbf{1 8}$ & $4-\mathrm{CF} 3$ & $4-\mathrm{SO} 2 \mathrm{Me}$ & $\mathrm{Me}$ & $\mathrm{H}$ & 7.1 \\
\hline $\mathbf{1 9}$ & $4-\mathrm{Me}$ & $4-\mathrm{SO} 2 \mathrm{Me}$ & $\mathrm{Me}$ & $\mathrm{H}$ & 7.4 \\
\hline $\mathbf{2 0}$ & $4-\mathrm{COCH} 3$ & $4-\mathrm{SO} 2 \mathrm{Me}$ & $\mathrm{Me}$ & $\mathrm{H}$ & 5.54 \\
\hline $\mathbf{2 1}$ & $3,4-\mathrm{diF}$ & $4-\mathrm{SO} 2 \mathrm{Me}$ & $\mathrm{Me}$ & $\mathrm{H}$ & 6.6 \\
\hline
\end{tabular}

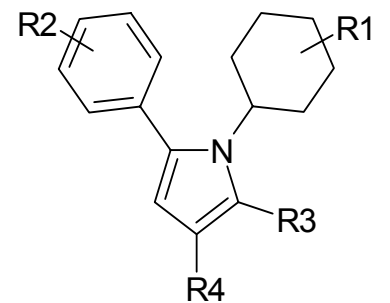

\begin{tabular}{|c|c|c|c|c|c|}
\hline Compound & R1 & R2 & R3 & R4 & pIC $_{\mathbf{5 0}}$ \\
\hline $\mathbf{2 2}$ & $\mathrm{H}$ & $4-\mathrm{SO} 2 \mathrm{Me}$ & $\mathrm{Me}$ & $\mathrm{H}$ & 6.28 \\
\hline
\end{tabular}


Table II : Structures and COX-2 inhibition power of imidazole derivatives.

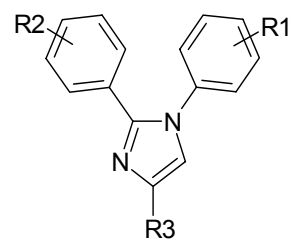

\begin{tabular}{|c|c|c|c|c|}
\hline Compound & R1 & R2 & R3 & $\mathrm{pIC}_{50}$ \\
\hline 23 & $4-\mathrm{SO} 2 \mathrm{Me}$ & $4-\mathrm{Cl}$ & $\mathrm{CH} 2 \mathrm{SCH} 3$ & 6.49 \\
\hline 24 & $4-\mathrm{SO} 2 \mathrm{Me}$ & $4-\mathrm{Cl}$ & $\mathrm{CH} 2 \mathrm{CN}$ & 5.81 \\
\hline 25 & 4-SO2Me & $\mathrm{H}$ & CF3 & 6.92 \\
\hline 26 & $4-\mathrm{SO} 2 \mathrm{Me}$ & $\mathrm{Me}$ & CF3 & 6.80 \\
\hline 27 & 4-SO2Me & $\mathrm{OMe}$ & CF3 & 6.24 \\
\hline 28 & $4-\mathrm{SO} 2 \mathrm{Me}$ & $\mathrm{NMe}$ & CF3 & 5.83 \\
\hline 29 & $4-\mathrm{SO} 2 \mathrm{Me}$ & $\mathrm{N}(\mathrm{Me})_{2}$ & CF3 & 6.15 \\
\hline 30 & $4-\mathrm{SO} 2 \mathrm{Me}$ & $\mathrm{SMe}$ & CF3 & 6.80 \\
\hline 31 & $4-\mathrm{SO} 2 \mathrm{Me}$ & $4-\mathrm{SO} 2 \mathrm{Me}$ & CF3 & 5.24 \\
\hline 32 & 4-SO2NH2 & 4-Cl & CF3 & 8.00 \\
\hline 33 & 4-SO2NH2 & $4-\mathrm{F}$ & CF3 & 8.00 \\
\hline 34 & 4-SO2NH2 & $\mathrm{H}$ & CF3 & 7.40 \\
\hline 35 & 4-SO2NH2 & $\mathrm{Me}$ & CF3 & 7.40 \\
\hline 36 & $4-\mathrm{SO} 2 \mathrm{Me}$ & $3-\mathrm{Cl}$ & CF3 & 7.22 \\
\hline 37 & $4-\mathrm{SO} 2 \mathrm{Me}$ & $3-\mathrm{F}$ & CF3 & 6.92 \\
\hline 38 & 4-SO2Me & $3-\mathrm{Br}$ & CF3 & 7.10 \\
\hline 39 & $4-\mathrm{SO} 2 \mathrm{Me}$ & $3-\mathrm{Me}$ & CF3 & 7.22 \\
\hline 40 & $4-\mathrm{SO} 2 \mathrm{Me}$ & $3-\mathrm{CF} 3$ & CF3 & 6.68 \\
\hline 41 & 4-SO2Me & 3-OMe & CF3 & 6.46 \\
\hline 42 & 4-SO2Me & 3-SMe & CF3 & 6.46 \\
\hline 43 & 4-SO2Me & $3-\mathrm{CH} 2 \mathrm{OMe}$ & CF3 & 4.17 \\
\hline 44 & 4-SO2Me & $3-\mathrm{N}(\mathrm{Me})_{2}$ & CF3 & 5.49 \\
\hline 45 & $4-\mathrm{SO} 2 \mathrm{Me}$ & 3-NHMe & CF3 & 6.04 \\
\hline 46 & $4-\mathrm{SO} 2 \mathrm{Me}$ & $3-\mathrm{NH} 2$ & CF3 & 5.23 \\
\hline 47 & 4-SO2Me & $3-\mathrm{NO} 2$ & CF3 & 6.24 \\
\hline 48 & 4-SO2NH2 & $3-\mathrm{Cl}$ & CF3 & 8.10 \\
\hline 49 & 4-SO2NH2 & $3-\mathrm{F}$ & CF3 & 7.52 \\
\hline 50 & 4-SO2NH2 & $3-\mathrm{Br}$ & CF3 & 8.15 \\
\hline 51 & 4-SO2NH2 & $3-\mathrm{Me}$ & CF3 & 7.52 \\
\hline 52 & 4-SO2Me & $2-\mathrm{Cl}$ & CF3 & 6.05 \\
\hline 53 & $4-\mathrm{SO} 2 \mathrm{Me}$ & $2-\mathrm{F}$ & CF3 & 6.40 \\
\hline 54 & $4-\mathrm{SO} 2 \mathrm{Me}$ & $2-\mathrm{Me}$ & CF3 & 6.10 \\
\hline 55 & 4-SO2Me & $2-\mathrm{OMe}$ & CF3 & 4.00 \\
\hline 56 & 4-SO2NH2 & $2-\mathrm{F}$ & CF3 & 7.00 \\
\hline 57 & 4-SO2NH2 & $2-\mathrm{Me}$ & CF3 & 6.70 \\
\hline 58 & 4-SO2Me & 4-OMe-3-F & CF3 & 6.82 \\
\hline
\end{tabular}




\begin{tabular}{|c|c|c|c|c|}
\hline 59 & 4-SO2Me & 4-OMe-3-Cl & CF3 & 6.89 \\
\hline 60 & 4-SO2Me & 4-SMe-3-Cl & CF3 & 7.40 \\
\hline 61 & 4-SO2Me & $4-\mathrm{N}(\mathrm{Me})_{2}-3-\mathrm{Cl}$ & CF3 & 6.49 \\
\hline 62 & 4-SO2Me & $4-\mathrm{N}(\mathrm{Me})_{2}-3-\mathrm{F}$ & CF3 & 6.48 \\
\hline 63 & 4-SO2Me & 4-NHMe-3-Cl & CF3 & 6.18 \\
\hline 64 & 4-SO2Me & 4-Me-3-Cl & CF3 & 7.52 \\
\hline 65 & $4-\mathrm{SO} 2 \mathrm{Me}$ & 4-Me-3-F & CF3 & 6.96 \\
\hline 66 & 4-SO2Me & 4-F-3-Me & CF3 & 6.77 \\
\hline 67 & 4-SO2Me & 4-Cl-3-Me & CF3 & 7.05 \\
\hline 68 & $4-\mathrm{SO} 2 \mathrm{Me}$ & 4-Cl-3-OMe & CF3 & 6.60 \\
\hline 69 & 4-SO2Me & $4-\mathrm{Cl}-3-\mathrm{N}(\mathrm{Me})_{2}$ & CF3 & 5.98 \\
\hline 70 & 4-SO2Me & 3,4-diF & CF3 & 6.92 \\
\hline 71 & 4-SO2Me & 3,4-diMe & CF3 & 6.48 \\
\hline 72 & 4-SO2Me & 3-Cl-5-Me & CF3 & 7.10 \\
\hline 73 & 4-SO2Me & $4-\mathrm{Cl}$ & $\mathrm{Me}$ & 6.62 \\
\hline 74 & 4-SO2Me & 3-F-5-Me & CF3 & 6.96 \\
\hline 75 & $4-\mathrm{SO} 2 \mathrm{Me}$ & 3-F-5-OMe & CF3 & 6.02 \\
\hline 76 & 4-SO2Me & 3,5-diCl & CF3 & 6.77 \\
\hline 77 & 4-SO2NH2 & 3-F-4-OMe & CF3 & 7.52 \\
\hline 78 & 4-SO2NH2 & 3-Cl-4-OMe & CF3 & 7.70 \\
\hline 79 & 4-SO2NH2 & 3-Br-4-OMe & CF3 & 7.52 \\
\hline 80 & 4-SO2NH2 & 3-Cl-4-SMe & CF3 & 8.00 \\
\hline 81 & 4-SO2Me & $4-\mathrm{Cl}$ & CF3 & 6.96 \\
\hline 82 & 4-SO2NH2 & 3-Cl-4-Me & CF3 & 8.52 \\
\hline 83 & 4-SO2NH2 & 4-Cl-3-OMe & CF3 & 7.70 \\
\hline 84 & 4-SO2NH2 & 3,4-diF & CF3 & 7.52 \\
\hline 85 & 4-SO2NH2 & 3-Cl-5-Me & CF3 & 7.40 \\
\hline 86 & 4-SO2NH2 & $3-\mathrm{F}-5-\mathrm{Me}$ & CF3 & 7.52 \\
\hline 87 & 4-SO2NH2 & 3-F-5-OMe & CF3 & 6.34 \\
\hline 88 & 4-SO2Me & 3,5-diF-4-OMe & CF3 & 6.77 \\
\hline 89 & $4-\mathrm{SO} 2 \mathrm{Me}$ & 3,5-diCl-4-OMe & CF3 & 6.85 \\
\hline 90 & $4-\mathrm{SO} 2 \mathrm{Me}$ & 3,5-diBr-4-OMe & CF3 & 7.05 \\
\hline 91 & 4-SO2Me & 3,5-diMe-4-OMe & CF3 & 6.14 \\
\hline 92 & 4-SO2Me & 2,5-diMe-4-OMe & CF3 & 4.91 \\
\hline 93 & 4-SO2Me & 3,5-diCl-4-N(Me $)_{2}$ & CF3 & 6.85 \\
\hline 94 & 4-SO2NH2 & 3,5-diF-4-OMe & CF3 & 7.52 \\
\hline 95 & 4-SO2Me & $4-\mathrm{Cl}$ & CHF2 & 6.21 \\
\hline 96 & $4-\mathrm{SO} 2 \mathrm{Me}$ & $4-\mathrm{Cl}$ & $\mathrm{CH} 2 \mathrm{~F}$ & 6.39 \\
\hline 97 & 4-SO2Me & $4-\mathrm{Cl}$ & $\mathrm{CHO}$ & 5.80 \\
\hline 98 & 4-SO2Me & $4-\mathrm{Cl}$ & COOEt & 5.24 \\
\hline 99 & $4-\mathrm{SO} 2 \mathrm{Me}$ & $4-\mathrm{Cl}$ & $\mathrm{CN}$ & 6.64 \\
\hline 100 & 4-SO2Me & $4-\mathrm{Cl}$ & $\mathrm{Ph}$ & 6.62 \\
\hline 101 & 4-SO2Me & $4-\mathrm{Cl}$ & $\mathrm{CH} 2 \mathrm{OMe}$ & 5.43 \\
\hline 102 & 4-SO2Me & $4-\mathrm{F}$ & CF3 & 7.00 \\
\hline 103 & 4-F & 4-SO2Me & CF3 & 5.23 \\
\hline
\end{tabular}




\begin{tabular}{|l|l|l|l|l|}
\hline $\mathbf{1 0 4}$ & 4-Cl & 4-SO2Me & $\mathrm{CH} 2 \mathrm{OH}$ & 5.08 \\
\hline
\end{tabular}

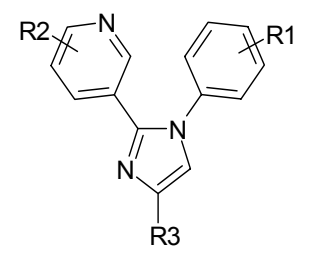

\begin{tabular}{|c|c|c|c|c|}
\hline Compound & $\mathbf{R} 1$ & $\mathbf{R 2}$ & $\mathbf{R 3}$ & $\mathbf{p I C}_{\mathbf{5 0}}$ \\
\hline $\mathbf{1 0 5}$ & $4-\mathrm{SO} 2 \mathrm{Me}$ & $\mathrm{H}$ & $\mathrm{CF} 3$ & 5.77 \\
\hline $\mathbf{1 0 6}$ & $4-\mathrm{SO} 2 \mathrm{Me}$ & $2-\mathrm{Me}$ & $\mathrm{CF} 3$ & 5.02 \\
\hline $\mathbf{1 0 7}$ & $4-\mathrm{SO} 2 \mathrm{Me}$ & $6-\mathrm{Me}$ & $\mathrm{CF} 3$ & 5.74 \\
\hline $\mathbf{1 0 8}$ & $4-\mathrm{SO} 2 \mathrm{Me}$ & $5-\mathrm{Me}$ & $\mathrm{CF} 3$ & 5.74 \\
\hline $\mathbf{1 0 9}$ & $4-\mathrm{SO} 2 \mathrm{Me}$ & $4-\mathrm{Me}$ & $\mathrm{CF} 3$ & 4.27 \\
\hline $\mathbf{1 1 0}$ & $4-\mathrm{SO} 2 \mathrm{Me}$ & $6-\mathrm{OMe}$ & $\mathrm{CF} 3$ & 5.92 \\
\hline $\mathbf{1 1 1}$ & $4-\mathrm{SO} 2 \mathrm{Me}$ & $5-\mathrm{OMe}$ & $\mathrm{CF} 3$ & 4.42 \\
\hline $\mathbf{1 1 2}$ & $4-\mathrm{SO} 2 \mathrm{Me}$ & $5-\mathrm{Br}$ & $\mathrm{CF} 3$ & 6.02 \\
\hline $\mathbf{1 1 3}$ & $4-\mathrm{SO} 2 \mathrm{NH} 2$ & $\mathrm{H}$ & $\mathrm{CF} 3$ & 6.36 \\
\hline $\mathbf{1 1 4}$ & $4-\mathrm{SO} 2 \mathrm{NH} 2$ & $2-\mathrm{Me}$ & $\mathrm{CF} 3$ & 5.55 \\
\hline $\mathbf{1 1 5}$ & $4-\mathrm{SO} 2 \mathrm{NH} 2$ & $6-\mathrm{Me}$ & $\mathrm{CF} 3$ & 6.54 \\
\hline $\mathbf{1 1 6}$ & $4-\mathrm{SO} 2 \mathrm{NH} 2$ & $5-\mathrm{Me}$ & $\mathrm{CF} 3$ & 6.29 \\
\hline $\mathbf{1 1 7}$ & $4-\mathrm{SO} 2 \mathrm{NH} 2$ & $4-\mathrm{Me}$ & $\mathrm{CF} 3$ & 4.29 \\
\hline $\mathbf{1 1 8}$ & $4-\mathrm{SO} 2 \mathrm{NH} 2$ & $5-\mathrm{Br}$ & $\mathrm{CF} 3$ & 6.47 \\
\hline $\mathbf{1 1 9}$ & $4-\mathrm{SO} 2 \mathrm{CH} 3$ & $\mathrm{H}$ & $\mathrm{CHF}$ & 4.68 \\
\hline $\mathbf{1 2 0}$ & $4-\mathrm{SO} 2 \mathrm{CH} 3$ & $\mathrm{H}$ & $\mathrm{CN}$ & 4.61 \\
\hline $\mathbf{1 2 1}$ & $4-\mathrm{SO} 2 \mathrm{CH} 3$ & $\mathrm{H}$ & $\mathrm{CH} 3$ & 4.10 \\
\hline $\mathbf{1 2 2}$ & $4-\mathrm{SO} 2 \mathrm{NH} 2$ & $\mathrm{H}$ & $\mathrm{CHF} 2$ & 5.74 \\
\hline
\end{tabular}

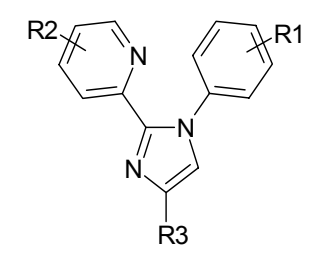

\begin{tabular}{|c|c|c|c|c|}
\hline Compound & R1 & R2 & R3 & pIC $_{\mathbf{5 0}}$ \\
\hline $\mathbf{1 2 3}$ & 4-SO2Me & H & CF3 & 5.82 \\
\hline $\mathbf{1 2 4}$ & 4-SO2Me & 3-Me & CF3 & 5.54 \\
\hline $\mathbf{1 2 5}$ & 4-SO2Me & 4-Me & CF3 & 5.89 \\
\hline $\mathbf{1 2 6}$ & $4-S O 2 M e$ & $5-M e$ & CF3 & 6.28 \\
\hline $\mathbf{1 2 7}$ & 4-SO2Me & 6-Me & CF3 & 5.24 \\
\hline $\mathbf{1 2 8}$ & 4-SO2NH2 & 3-Me & CF3 & 6.38 \\
\hline
\end{tabular}


- Page 7 -

\begin{tabular}{|l|l|l|l|l|}
\hline $\mathbf{1 2 9}$ & $4-\mathrm{SO} 2 \mathrm{NH} 2$ & $4-\mathrm{Me}$ & CF3 & 6.14 \\
\hline $\mathbf{1 3 0}$ & $4-\mathrm{SO} 2 \mathrm{NH} 2$ & $5-\mathrm{Me}$ & CF3 & 6.36 \\
\hline $\mathbf{1 3 1}$ & $4-\mathrm{SO} 2 \mathrm{NH} 2$ & $6-\mathrm{Me}$ & CF3 & 5.80 \\
\hline
\end{tabular}

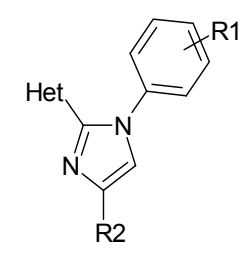

\begin{tabular}{|c|c|c|c|c|}
\hline Compound & R1 & $\mathbf{R 2}$ & Het & $\mathrm{pIC}_{50}$ \\
\hline 132 & 4-SO2Me & CF3 & & 5.92 \\
\hline 133 & 4-SO2Me & CF3 & & 5.77 \\
\hline 134 & 4-SO2Me & CF3 & & 6.20 \\
\hline 135 & 4-SO2Me & CF3 & & 5.96 \\
\hline 136 & 4-SO2Me & CF3 & & 6.55 \\
\hline 137 & 4-SO2Me & CF3 & & 6.33 \\
\hline
\end{tabular}


- Page 8 -

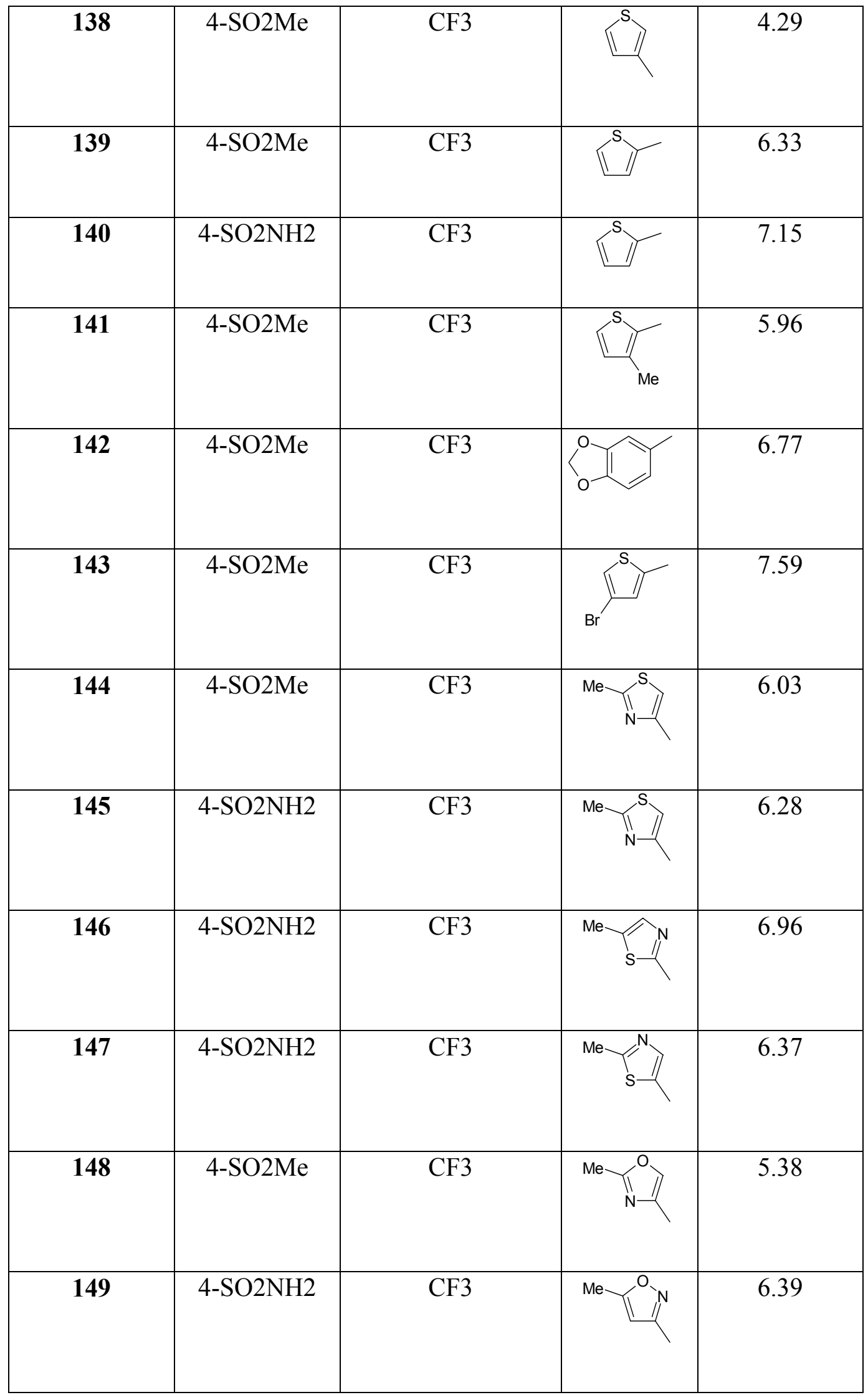


Table III : Structures and COX-2 inhibition power of cyclopentene derivatives.

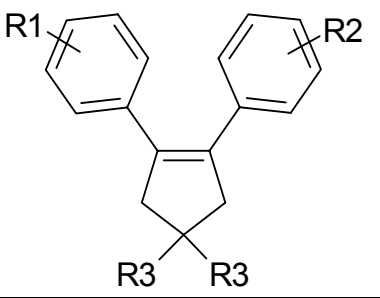

\begin{tabular}{|c|c|c|c|c|}
\hline Compound & R1 & $\mathbf{R 2}$ & R3 & $\mathrm{pIC}_{50}$ \\
\hline 150 & 4-SO2Me & 3,4-diF & $\mathrm{H}$ & 7.29 \\
\hline 151 & 4-SO2Me & $4-\mathrm{F}-3-\mathrm{Cl}$ & $\mathrm{H}$ & 7.52 \\
\hline 152 & 4-SO2Me & 3,4-diCl & $\mathrm{H}$ & 8.00 \\
\hline 153 & 4-SO2Me & 4-OMe-3-F & $\mathrm{H}$ & 6.92 \\
\hline 154 & $4-\mathrm{SO} 2 \mathrm{Me}$ & 4-OMe-3-Cl & $\mathrm{H}$ & 6.85 \\
\hline 155 & 4-SO2Me & 4-OMe-3,5-diCl & $\mathrm{H}$ & 7.77 \\
\hline 156 & 4-SO2Me & $3,4-\mathrm{OCH} 2 \mathrm{CH} 2 \mathrm{O}$ & $\mathrm{H}$ & 7.68 \\
\hline 157 & 4-SO2Me & $4-\mathrm{NMe}_{2}-3-\mathrm{Cl}$ & $\mathrm{H}$ & 8.30 \\
\hline 158 & 4-SO2Me & 4-CF3-3-F & $\mathrm{H}$ & 6.12 \\
\hline 159 & $4-\mathrm{SO} 2 \mathrm{NH} 2$ & $4-\mathrm{F}$ & $\mathrm{H}$ & 8.15 \\
\hline 160 & 4-SO2NH2 & 3,4-diF & $\mathrm{H}$ & 7.74 \\
\hline 161 & 4-SO2NH2 & $4-\mathrm{F}-3-\mathrm{Cl}$ & $\mathrm{H}$ & 8.00 \\
\hline 162 & 4-SO2NH2 & 3,4,5-triF & $\mathrm{H}$ & 5.54 \\
\hline 163 & 4-SO2NH2 & $4-\mathrm{Cl}$ & $\mathrm{H}$ & 8.52 \\
\hline 164 & $4-\mathrm{SO} 2 \mathrm{NH} 2$ & 3,4-diCl & $\mathrm{H}$ & 8.70 \\
\hline 165 & 4-SO2NH2 & 4-OMe & $\mathrm{H}$ & 8.70 \\
\hline 166 & 4-SO2NH2 & 3-F-4-OMe & $\mathrm{H}$ & 7.80 \\
\hline 167 & 4-SO2NH2 & 3-Cl-4-OMe & $\mathrm{H}$ & 8.05 \\
\hline 168 & $4-\mathrm{SO} 2 \mathrm{NH} 2$ & 3,5-diCl-4-OMe & $\mathrm{H}$ & 8.05 \\
\hline
\end{tabular}




\begin{tabular}{|c|c|c|c|c|}
\hline $\mathbf{1 6 9}$ & $4-\mathrm{SO} 2 \mathrm{NH} 2$ & $3,4-\mathrm{OCH} 2 \mathrm{CH} 2 \mathrm{O}$ & $\mathrm{H}$ & 8.70 \\
\hline $\mathbf{1 7 0}$ & $4-\mathrm{SO} 2 \mathrm{NH} 2$ & $4-\mathrm{Ne}_{2}-3-\mathrm{Cl}$ & $\mathrm{H}$ & 8.70 \\
\hline $\mathbf{1 7 1}$ & $4-\mathrm{SO} 2 \mathrm{NH} 2$ & $4-\mathrm{CF} 3$ & $\mathrm{H}$ & 6.82 \\
\hline $\mathbf{1 7 2}$ & $4-\mathrm{SO} 2 \mathrm{NH} 2$ & $3-\mathrm{F}-4-\mathrm{CF} 3$ & $\mathrm{H}$ & 6.77 \\
\hline $\mathbf{1 7 3}$ & $4-\mathrm{SO} 2 \mathrm{Me}$ & $4-\mathrm{F}$ & $\mathrm{H}$ & 7.59 \\
\hline $\mathbf{1 7 4}$ & $4-\mathrm{SO} 2 \mathrm{Me}$ & $4-\mathrm{OMe}$ & $\mathrm{H}$ & 8.30 \\
\hline $\mathbf{1 7 5}$ & $4-\mathrm{SO} 2 \mathrm{Me}$ & $4-\mathrm{Cl}$ & $\mathrm{H}$ & 8.52 \\
\hline $\mathbf{1 7 6}$ & $4-\mathrm{SO} 2 \mathrm{Me}$ & $4-\mathrm{Me}$ & $\mathrm{H}$ & 8.52 \\
\hline $\mathbf{1 7 7}$ & $4-\mathrm{SO} 2 \mathrm{Me}$ & $\mathrm{H}$ & $\mathrm{H}$ & 5.65 \\
\hline $\mathbf{1 7 8}$ & $4-\mathrm{SO} 2 \mathrm{Me}$ & $4-\mathrm{CF} 3$ & $\mathrm{H}$ & 6.06 \\
\hline $\mathbf{1 7 9}$ & $4-\mathrm{SO} 2 \mathrm{Me}$ & $2,4-\mathrm{diCl}$ & $\mathrm{H}$ & 7.28 \\
\hline $\mathbf{1 8 0}$ & $4-\mathrm{SO} 2 \mathrm{Me}$ & $4-\mathrm{CN}$ & $\mathrm{H}$ & 4.11 \\
\hline $\mathbf{1 8 1}$ & $4-\mathrm{SO} 2 \mathrm{Me}$ & $4-\mathrm{CH} 2 \mathrm{OH}$ & $\mathrm{H}$ & 5.49 \\
\hline $\mathbf{1 8 2}$ & $4-\mathrm{SO} 2 \mathrm{Me}$ & $4-\mathrm{CH} 2 \mathrm{OMe}$ & $\mathrm{H}$ & 5.18 \\
\hline $\mathbf{1 8 3}$ & $4-\mathrm{SO} 2 \mathrm{Me}$ & $4-\mathrm{SMe}$ & $\mathrm{H}$ & 6.66 \\
\hline $\mathbf{1 8 4}$ & $4-\mathrm{SO} 2 \mathrm{Me}$ & $2-\mathrm{Me}-4-\mathrm{F}$ & $\mathrm{H}$ & 7.12 \\
\hline $\mathbf{1 8 5}$ & $4-\mathrm{SO} 2 \mathrm{Me}$ & $4-\mathrm{F}$ & $\mathrm{Me}$ & 7.82 \\
\hline $\mathbf{1 8 6}$ & $4-\mathrm{SO} 2 \mathrm{Me}$ & $4-\mathrm{Cl}$ & $\mathrm{Me}$ & 8.15 \\
\hline $\mathbf{1 8 7}$ & $4-\mathrm{SO} 2 \mathrm{Me}$ & $4-\mathrm{F}$ & $\mathrm{CF} 3$ & 7.17 \\
\hline $\mathbf{1 8 8}$ & $4-\mathrm{SO} 2 \mathrm{Me}$ & $4-\mathrm{Cl}$ & $\mathrm{Et}$ & 4.19 \\
\hline $\mathbf{1 8 9}$ & $4-\mathrm{SO} 2 \mathrm{Me}$ & $4-\mathrm{F}$ & $\mathrm{CH} 2 \mathrm{~F}$ & 7.29 \\
\hline
\end{tabular}

Table IV: Structures and COX-2 inhibition power of benzene derivatives.

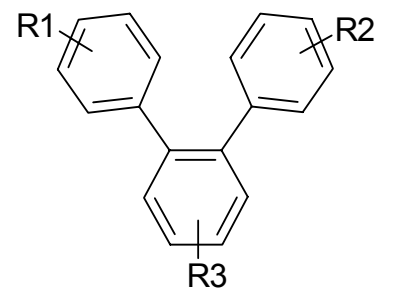

\begin{tabular}{|c|c|c|c|c|}
\hline Compound & R1 & $\mathbf{R 2}$ & R3 & $\mathrm{pIC}_{50}$ \\
\hline 190 & 4-SO2Me & $4-\mathrm{F}$ & $\mathrm{H}$ & 6.59 \\
\hline 191 & 4-SO2Me & $4-\mathrm{F}-3-\mathrm{Cl}$ & $\mathrm{H}$ & 6.44 \\
\hline 192 & 4-SO2Me & $3-\mathrm{Cl}$ & $\mathrm{H}$ & 6.85 \\
\hline 193 & 4-SO2Me & 4-OMe-3-Cl & $\mathrm{H}$ & 4.94 \\
\hline 194 & 4-SO2NH2 & $4-\mathrm{F}$ & $\mathrm{H}$ & 7.21 \\
\hline 195 & 4-SO2NH2 & $4-\mathrm{F}-3-\mathrm{Cl}$ & $\mathrm{H}$ & 7.77 \\
\hline 196 & 4-SO2NH2 & $3-\mathrm{Cl}$ & $\mathrm{H}$ & 8.22 \\
\hline 197 & 4-SO2NH2 & 4-OMe-3-F & $\mathrm{H}$ & 7.48 \\
\hline 198 & 4-SO2NH2 & 4-OMe-3-Cl & $\mathrm{H}$ & 7.72 \\
\hline 199 & $4-\mathrm{SO} 2 \mathrm{Me}$ & $4-\mathrm{F}$ & 3,4-diF & 7.85 \\
\hline 200 & 4-SO2Me & $4-\mathrm{F}-3-\mathrm{Cl}$ & 3,4-diF & 8.00 \\
\hline 201 & 4-SO2Me & 4-F-3-Me & 3,4-diF & 8.30 \\
\hline 202 & 4-SO2Me & 4-OMe-3-F & 3,4-diF & 7.68 \\
\hline 203 & 4-SO2Me & 4-OMe-3-Cl & 3,4-diF & 7.72 \\
\hline
\end{tabular}




\begin{tabular}{|c|c|c|c|c|}
\hline 204 & $4-\mathrm{SO} 2 \mathrm{Me}$ & 4-OMe-3-OMe & 3,4-diF & 6.47 \\
\hline 205 & 4-SO2Me & 3,4-OCH2CH2O & 3,4-diF & 6.47 \\
\hline 206 & $4-\mathrm{SO} 2 \mathrm{Me}$ & $3,4-\mathrm{OCH} 2 \mathrm{O}$ & $3,4-\mathrm{diF}$ & 7.92 \\
\hline 207 & $4-\mathrm{SO} 2 \mathrm{Me}$ & $4-\mathrm{Me}$ & 3,4-diF & 8.15 \\
\hline 208 & 4-SO2Me & 4-Me-3-Cl & 3,4-diF & 7.89 \\
\hline 209 & 4-SO2Me & 3,4-diMe & 3,4-diF & 7.64 \\
\hline 210 & $4-\mathrm{SO} 2 \mathrm{Me}$ & 4-Cl-3-Me & 3,4-diF & 8.22 \\
\hline 211 & 4-SO2Me & $4-\mathrm{NMe}_{2}-3-\mathrm{Cl}$ & 3,4-diF & 8.10 \\
\hline 212 & 4-SO2NH2 & $4-\mathrm{F}$ & 3,4-diF & 8.40 \\
\hline 213 & $4-\mathrm{SO} 2 \mathrm{NH} 2$ & $3-\mathrm{Cl}-4-\mathrm{F}$ & 3,4-diF & 8.70 \\
\hline 214 & 4-SO2NH2 & 3-Me-4-F & 3,4-diF & 8.70 \\
\hline 215 & 4-SO2NH2 & 3-F-4-OMe & 3,4-diF & 7.89 \\
\hline 216 & 4-SO2NH2 & 3-Cl-4-OMe & 3,4-diF & 7.89 \\
\hline 217 & 4-SO2NH2 & 3,5-diCl-4-OMe & 3,4-diF & 7.68 \\
\hline 218 & $4-\mathrm{SO} 2 \mathrm{NH} 2$ & 3-Me-4-OMe & 3,4-diF & 8.30 \\
\hline 219 & 4-SO2NH2 & 3,4-diOMe & 3,4-diF & 7.19 \\
\hline 220 & 4-SO2NH2 & 3,4-OCH2CH2O & 3,4-diF & 7.49 \\
\hline 221 & 4-SO2NH2 & $3,4-\mathrm{OCH} 2 \mathrm{O}$ & 3,4-diF & 8.40 \\
\hline 222 & 4-SO2NH2 & 4-Me & 3,4-diF & 8.40 \\
\hline 223 & 4-SO2NH2 & 3-Cl-4-Me & 3,4-diF & 8.52 \\
\hline 224 & 4-SO2NH2 & 3-Cl-4-Me & 3,4-diF & 8.30 \\
\hline 225 & 4-SO2NH2 & 3-Me-4-Cl & 3,4-diF & 8.52 \\
\hline 226 & 4-SO2NH2 & $4-\mathrm{NMe}_{2}-3-\mathrm{Cl}$ & 3,4-diF & 8.22 \\
\hline 227 & 4-SO2Me & $4-\mathrm{F}$ & 3,4-diCl & 6.62 \\
\hline 228 & 4-SO2Me & $4-\mathrm{F}$ & $3,4-\mathrm{OCH} 2 \mathrm{O}$ & 7.08 \\
\hline 229 & 4-SO2Me & 3-Me-4-OMe & 3,4-diF & 7.89 \\
\hline
\end{tabular}

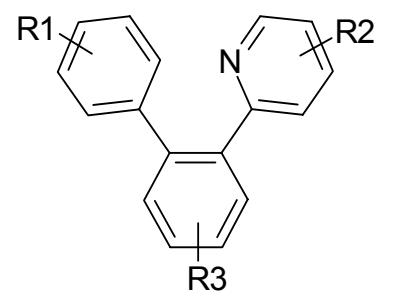

\begin{tabular}{|c|c|c|c|c|}
\hline Compound & R1 & R2 & R3 & pIC $_{\mathbf{5 0}}$ \\
\hline $\mathbf{2 3 0}$ & $4-\mathrm{SO} 2 \mathrm{Me}$ & $4-\mathrm{Me}$ & $3,4-\mathrm{diF}$ & 4.28 \\
\hline $\mathbf{2 3 1}$ & $4-\mathrm{SO} 2 \mathrm{NH} 2$ & $4-\mathrm{Me}$ & $3,4-\mathrm{diF}$ & 6.48 \\
\hline
\end{tabular}

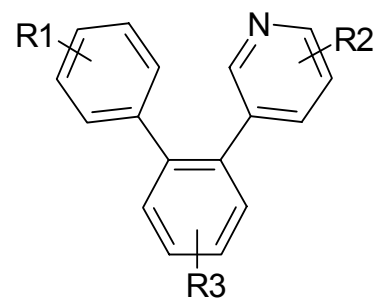




\begin{tabular}{|c|c|c|c|c|}
\hline Compound & R1 & R2 & R3 & pIC $_{\mathbf{5 0}}$ \\
\hline $\mathbf{2 3 2}$ & $4-\mathrm{SO} 2 \mathrm{Me}$ & $4-\mathrm{Me}$ & $3,4-\mathrm{diF}$ & 6.77 \\
\hline $\mathbf{2 3 3}$ & $4-\mathrm{SO} 2 \mathrm{NH} 2$ & $4-\mathrm{Me}$ & $3,4-\mathrm{diF}$ & 7.29 \\
\hline
\end{tabular}

Table V: Structures and COX-2 inhibition power of pyrazole derivatives.

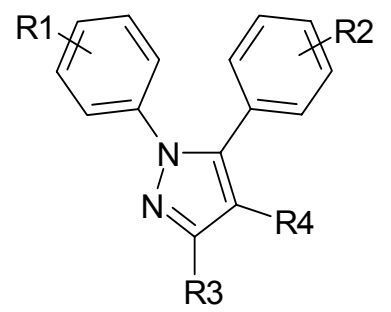

\begin{tabular}{|c|c|c|c|c|c|}
\hline Compound & R1 & R2 & R3 & R4 & pIC $_{\mathbf{5 0}}$ \\
\hline $\mathbf{2 3 4}$ & $4-\mathrm{SO} 2 \mathrm{NH} 2$ & $4-\mathrm{F}$ & $\mathrm{CN}$ & $\mathrm{H}$ & 6.47 \\
\hline $\mathbf{2 3 5}$ & 4-SO2NH2 & $4-\mathrm{Cl}$ & $\mathrm{CH} 2 \mathrm{OH}$ & $\mathrm{H}$ & 6.08 \\
\hline $\mathbf{2 3 6}$ & 4-SO2NH2 & $\mathrm{H}$ & $\mathrm{CH} 2 \mathrm{~F}$ & $\mathrm{H}$ & 6.70 \\
\hline $\mathbf{2 3 7}$ & $4-\mathrm{SO} 2 \mathrm{NH} 2$ & $4-\mathrm{Cl}$ & $\mathrm{CH} 2 \mathrm{CN}$ & $\mathrm{H}$ & 6.92 \\
\hline
\end{tabular}




\begin{tabular}{|c|c|c|c|c|c|}
\hline 238 & 4-SO2NH2 & 3-Me-4-SMe & CF3 & $\mathrm{H}$ & 8.43 \\
\hline 239 & 4-SO2NH2 & 3-Cl-4-NMe 2 & CF3 & $\mathrm{H}$ & 8.24 \\
\hline 240 & 4-SO2NH2 & 3-Cl-4-NHMe & CF3 & $\mathrm{H}$ & 7.57 \\
\hline 241 & 4-SO2NH2 & 3-Cl-4-OMe-5-Me & CF3 & $\mathrm{H}$ & 7.18 \\
\hline 242 & 4-SO2NH2 & 3,4-diCl & CF3 & $\mathrm{H}$ & 7.82 \\
\hline 243 & 4-SO2NH2 & 2,4-diCl & CF3 & $\mathrm{H}$ & 7.25 \\
\hline 244 & 4-SO2NH2 & 2,4-diMe & CF3 & $\mathrm{H}$ & 6.92 \\
\hline 245 & 4-SO2NH2 & $\mathrm{H}$ & CF3 & $\mathrm{H}$ & 7.49 \\
\hline 246 & 4-SO2NH2 & $2-\mathrm{F}$ & CF3 & $\mathrm{H}$ & 7.24 \\
\hline 247 & 4-SO2NH2 & $3-\mathrm{F}$ & CF3 & $\mathrm{H}$ & 5.11 \\
\hline 248 & 4-SO2NH2 & $4-\mathrm{F}$ & CF3 & $\mathrm{H}$ & 7.39 \\
\hline 249 & 4-SO2NH2 & $2-\mathrm{Cl}$ & CF3 & $\mathrm{H}$ & 7.25 \\
\hline 250 & 4-SO2NH2 & 4-Cl & CF3 & $\mathrm{H}$ & 8.00 \\
\hline 251 & 4-SO2NH2 & 2-Me & CF3 & $\mathrm{H}$ & 7.16 \\
\hline 252 & 4-SO2NH2 & $3-\mathrm{Me}$ & CF3 & $\mathrm{H}$ & 6.96 \\
\hline 253 & 4-SO2NH2 & 4-Et & CF3 & $\mathrm{H}$ & 6.07 \\
\hline 254 & 4-SO2NH2 & 4-CF3 & CF3 & $\mathrm{H}$ & 5.08 \\
\hline 255 & 4-SO2NH2 & $4-\mathrm{NO} 2$ & CF3 & $\mathrm{H}$ & 5.58 \\
\hline 256 & 4-SO2NH2 & $2-\mathrm{OMe}$ & CF3 & $\mathrm{H}$ & 6.54 \\
\hline 257 & 4-SO2NH2 & 4-OMe & CF3 & $\mathrm{H}$ & 8.10 \\
\hline 258 & 4-SO2NH2 & 4-OEt & CF3 & $\mathrm{H}$ & 6.19 \\
\hline 259 & 4-SO2NH2 & 4-SMe & CF3 & $\mathrm{H}$ & 8.05 \\
\hline 260 & 4-SO2NH2 & 4-NH2 & CF3 & $\mathrm{H}$ & 6.47 \\
\hline 261 & 4-SO2NH2 & $2-\mathrm{NMe}_{2}$ & CF3 & $\mathrm{H}$ & 4.84 \\
\hline 262 & 4-SO2NH2 & 4-NHMe & CF3 & $\mathrm{H}$ & 7.80 \\
\hline 263 & 4-SO2NH2 & $4-\mathrm{NMe}_{2}$ & CF3 & $\mathrm{H}$ & 8.33 \\
\hline 264 & 4-SO2NH2 & $4-\mathrm{CH} 2 \mathrm{OH}$ & CF3 & $\mathrm{H}$ & 4.03 \\
\hline 265 & 4-SO2NH2 & 4-COOH & CF3 & $\mathrm{H}$ & 4.95 \\
\hline 266 & 4-SO2NH2 & 3-Me-4-OMe & CF3 & $\mathrm{H}$ & 8.03 \\
\hline 267 & 4-SO2NH2 & 3-Et-4-OMe & CF3 & $\mathrm{H}$ & 6.37 \\
\hline 268 & 4-SO2NH2 & 3,4-diOMe & CF3 & $\mathrm{H}$ & 6.22 \\
\hline 269 & 4-SO2NH2 & $4-\mathrm{Cl}$ & CF3 & $\mathrm{Cl}$ & 8.28 \\
\hline 270 & 4-SO2NH2 & $\mathrm{H}$ & $\mathrm{Me}$ & $\mathrm{Cl}$ & 7.55 \\
\hline 271 & 4-SO2NH2 & $4-\mathrm{Cl}$ & $\mathrm{CH} 2 \mathrm{OH}$ & $\mathrm{Cl}$ & 6.47 \\
\hline 272 & 4-SO2NH2 & $4-\mathrm{Cl}$ & $\mathrm{CN}$ & $\mathrm{Cl}$ & 8.00 \\
\hline 273 & 4-SO2NH2 & $4-\mathrm{Cl}$ & $\mathrm{COOH}$ & $\mathrm{Cl}$ & 4.15 \\
\hline 274 & 4-SO2NH2 & $4-\mathrm{Cl}$ & COOMe & $\mathrm{Cl}$ & 6.80 \\
\hline 275 & 4-SO2NH2 & $4-\mathrm{Cl}$ & COONH2 & $\mathrm{Cl}$ & 5.96 \\
\hline 276 & 4-SO2NH2 & $\mathrm{H}$ & $\mathrm{H}$ & $\mathrm{Cl}$ & 7.31 \\
\hline 277 & 4-SO2NH2 & $4-\mathrm{Cl}$ & $\mathrm{H}$ & $\mathrm{Br}$ & 7.51 \\
\hline 278 & 4-SO2NH2 & $\mathrm{H}$ & $\mathrm{H}$ & $\mathrm{F}$ & 5.33 \\
\hline 279 & 4-SO2NH2 & $\mathrm{H}$ & $\mathrm{H}$ & $\mathrm{Me}$ & 4.33 \\
\hline 280 & 4-SO2NH2 & 4-Me & $\mathrm{H}$ & $\mathrm{CN}$ & 7.12 \\
\hline 281 & 4-SO2NH2 & $\mathrm{H}$ & $\mathrm{H}$ & $\mathrm{NO} 2$ & 6.54 \\
\hline 282 & 4-SO2NH2 & $\mathrm{H}$ & $\mathrm{H}$ & $\mathrm{NH} 2$ & 4.53 \\
\hline
\end{tabular}




\begin{tabular}{|c|c|c|c|c|c|}
\hline 283 & 4-SO2NH2 & $\mathrm{H}$ & CF3 & $\mathrm{F}$ & 8.77 \\
\hline 284 & 4-SO2NH2 & $4-\mathrm{Cl}$ & CF3 & $\mathrm{Me}$ & 7.66 \\
\hline 285 & 4-SO2NH2 & $4-\mathrm{Cl}$ & CF3 & $\mathrm{Et}$ & 7.55 \\
\hline 286 & 4-SO2NH2 & $\mathrm{H}$ & CF3 & $\mathrm{OMe}$ & 7.10 \\
\hline 287 & 4-SO2NH2 & $\mathrm{H}$ & CF3 & $\mathrm{OH}$ & 5.45 \\
\hline 288 & $4-\mathrm{F}$ & 4-SO2NH2 & CF3 & $\mathrm{H}$ & 8.00 \\
\hline 289 & 4-OME & 4-SO2NH2 & CF3 & $\mathrm{H}$ & 8.17 \\
\hline 290 & 4-SO2NH2 & $4-\mathrm{Cl}$ & CHF2 & $\mathrm{H}$ & 8.00 \\
\hline 291 & 4-SO2NH2 & 4-Me & CHF2 & $\mathrm{H}$ & 7.89 \\
\hline 292 & 4-SO2NH2 & $4-\mathrm{CN}$ & CHF2 & $\mathrm{H}$ & 4.53 \\
\hline 293 & 4-SO2NH2 & 4-OMe & CHF2 & $\mathrm{H}$ & 7.82 \\
\hline 294 & 4-SO2NH2 & 3-F,4-OMe & CF3 & $\mathrm{H}$ & 7.30 \\
\hline 295 & 4-SO2NH2 & 3-Cl,4-OMe & CF3 & $\mathrm{H}$ & 7.57 \\
\hline 296 & 4-SO2NH2 & 3,5-diCl,4-OMe & CHF2 & $\mathrm{H}$ & 7.68 \\
\hline 297 & 4-SO2NH2 & 3,5-diF,4-OMe & CHF2 & $\mathrm{H}$ & 6.46 \\
\hline 298 & 4-SO2NH2 & $\mathrm{H}$ & CHF2 & $\mathrm{H}$ & 6.89 \\
\hline 299 & 4-Cl & 4-Cl & CF3 & $\mathrm{H}$ & 5.32 \\
\hline 300 & 4-OMe & 4-OMe & CF3 & $\mathrm{H}$ & 6.12 \\
\hline 301 & 4-Cl & 4-OMe & CF3 & $\mathrm{H}$ & 6.12 \\
\hline 302 & 4-OMe & 4-Cl & CF3 & $\mathrm{H}$ & 4.13 \\
\hline 303 & 4-SO2NH2 & $\mathrm{H}$ & $\mathrm{Me}$ & $\mathrm{H}$ & 4.20 \\
\hline 304 & 4-SO2Me & $4-\mathrm{F}$ & CF3 & $\mathrm{H}$ & 7.00 \\
\hline 305 & 4-SO2NH2 & 4-Me & CF3 & $\mathrm{H}$ & 7.40 \\
\hline
\end{tabular}

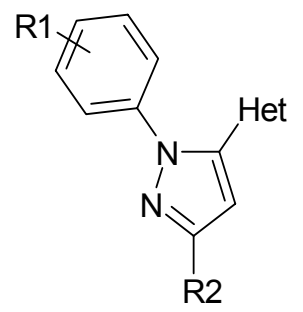


- Page 15 -

\begin{tabular}{|c|c|c|c|c|}
\hline Compound & Het & R1 & R2 & $\mathrm{pIC}_{50}$ \\
\hline 306 & & 4-SO2NH2 & $\mathrm{CF} 3$ & 4.34 \\
\hline 307 & & 4-SO2NH2 & $\mathrm{CF} 3$ & 4.19 \\
\hline 308 & & 4-SO2NH2 & CF3 & 7.92 \\
\hline 309 & & 4-SO2NH2 & CF3 & 7.59 \\
\hline 310 & & 4-SO2NH2 & CF3 & 6.46 \\
\hline 311 & & 4-SO2NH2 & CF3 & 4.35 \\
\hline 312 & & 4-SO2NH2 & CF3 & 7.08 \\
\hline 313 & & 4-SO2NH2 & CF3 & 7.51 \\
\hline 314 & & 4-SO2NH2 & CF3 & 6.64 \\
\hline 315 & & 4-SO2NH2 & CF3 & 6.05 \\
\hline 316 & & 4-SO2NH2 & CF3 & 7.68 \\
\hline
\end{tabular}


- Page 16 -

\begin{tabular}{|l|c|c|c|c|}
\hline 317 & 4-SO2NH2 & CF3 & 7.28 \\
\hline 318 & $y^{-\mathrm{Me}}$ & $4-\mathrm{SO} 2 \mathrm{NH} 2$ & $\mathrm{CHF} 2$ & 5.48 \\
\hline 319 & & & & \\
\hline & & & & \\
\hline
\end{tabular}


Table VI: Structures and COX-2 inhibition power of isoxazole derivatives.

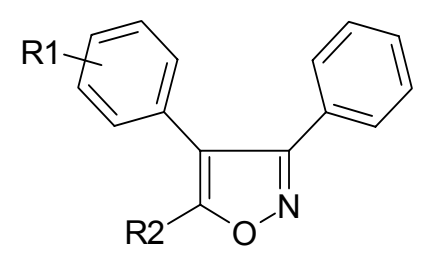

\begin{tabular}{|c|c|c|c|}
\hline Compound & R1 & R2 & pIC $_{\mathbf{5 0}}$ \\
\hline $\mathbf{3 2 0}$ & 4-SO2NH2 & $\mathrm{CH} 2 \mathrm{OH}$ & 6.74 \\
\hline $\mathbf{3 2 1}$ & 4-SO2NH2 & $\mathrm{Me}$ & 8.30 \\
\hline
\end{tabular}


Table VII: Structures and COX-2 inhibition power of spiroheptene derivatives.

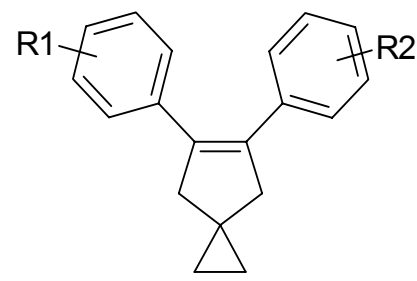

\begin{tabular}{|c|c|c|c|}
\hline Compound & R1 & $\mathbf{R 2}$ & $\mathrm{pIC}_{50}$ \\
\hline 322 & 4-SO2Me & 3-Br-4-OMe & 8.89 \\
\hline 323 & 4-SO2NH2 & 3-Br-4-OMe & 8.70 \\
\hline 324 & 4-SO2Me & 3,5-diCl-4-OMe & 8.22 \\
\hline 325 & 4-SO2NH2 & 3,5-diCl-4-OMe & 8.40 \\
\hline 326 & 4-SO2Me & 3-Cl-4-F & 8.15 \\
\hline 327 & 4-SO2NH2 & $3-\mathrm{Cl}-4-\mathrm{F}$ & 8.82 \\
\hline 328 & 4-SO2Me & 3-Cl-4-OMe & 7.77 \\
\hline 329 & 4-SO2NH2 & 3-Cl-4-OMe & 8.70 \\
\hline 330 & 4-SO2Me & 2,4-diCl & 7.48 \\
\hline 331 & 4-SO2Me & 4-Cl & 9.00 \\
\hline 332 & 4-SO2NH2 & 4-Cl & 9.00 \\
\hline 333 & 4-SO2Me & 3,4-diCl & 8.52 \\
\hline 334 & 4-SO2NH2 & 3,4-diCl & 9.00 \\
\hline 335 & 4-SO2Me & 4-Me & 8.82 \\
\hline 336 & 4-SO2Me & 3,4-diF & 8.48 \\
\hline 337 & 4-SO2Me & 4-F & 8.12 \\
\hline 338 & 4-SO2Me & 2,4-diF & 7.66 \\
\hline 339 & 4-SO2Me & 4-OCF3 & 6.87 \\
\hline 340 & 4-SO2Me & 3-F-4-OMe & 8.00 \\
\hline 341 & 4-SO2Me & $4-\mathrm{OMe}$ & 8.30 \\
\hline 342 & 4-SO2Me & 4-CF3 & 8.70 \\
\hline 343 & 4-SO2Me & $3,4-\mathrm{OCH} 2 \mathrm{O}$ & 8.60 \\
\hline 344 & 4-SO2NH2 & 3,4-diF & 8.52 \\
\hline 345 & 4-SO2NH2 & 4-F & 8.52 \\
\hline 346 & 4-SO2NH2 & 4-OCF3 & 6.89 \\
\hline 347 & 4-SO2NH2 & 3-F-4-OMe & 8.70 \\
\hline 348 & 4-SO2NH2 & 4-OMe & 9.00 \\
\hline 349 & 4-SO2NH2 & 4-CF3 & 9.00 \\
\hline
\end{tabular}

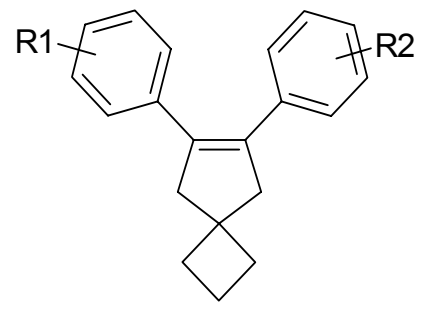




\begin{tabular}{|c|c|c|c|}
\hline Compound & R1 & R2 & pIC $_{\mathbf{5 0}}$ \\
\hline $\mathbf{3 5 0}$ & $4-\mathrm{SO} 2 \mathrm{Me}$ & $4-\mathrm{F}$ & 8.40 \\
\hline
\end{tabular}

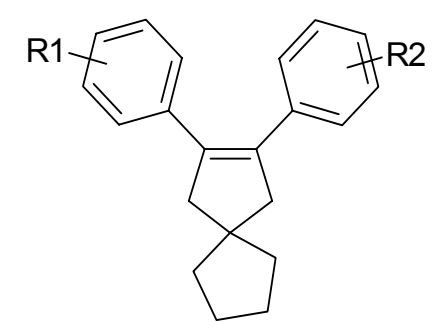

\begin{tabular}{|c|c|c|c|}
\hline Compound & R1 & R2 & pIC $_{\mathbf{5 0}}$ \\
\hline $\mathbf{3 5 1}$ & $4-\mathrm{SO} 2 \mathrm{Me}$ & $4-\mathrm{F}$ & 7.21 \\
\hline
\end{tabular}


Table VIII: Structures and COX-2 inhibition power of spiroheptadiene derivatives.

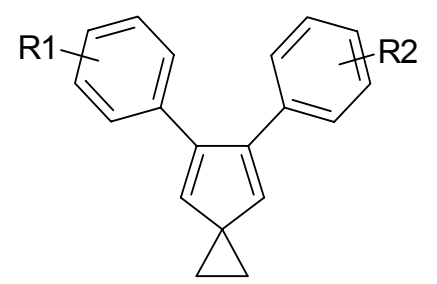

\begin{tabular}{|c|c|c|c|}
\hline Compound & R1 & R2 & pIC $_{\mathbf{5 0}}$ \\
\hline $\mathbf{3 5 2}$ & $4-\mathrm{SO} 2 \mathrm{Me}$ & $3-\mathrm{Cl}-4-\mathrm{OMe}$ & 7.21 \\
\hline $\mathbf{3 5 3}$ & $4-\mathrm{SO} 2 \mathrm{Me}$ & $4-\mathrm{F}$ & 7.59 \\
\hline
\end{tabular}

Table IX: Structures and COX-2 inhibition power of thiophene derivatives.

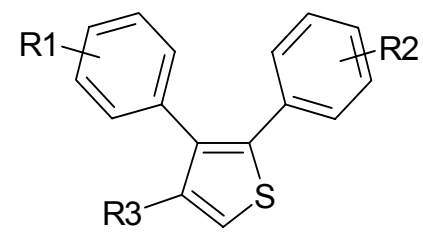

\begin{tabular}{|c|c|c|c|c|}
\hline Compound & R1 & R2 & R3 & pIC $_{\mathbf{5 0}}$ \\
\hline $\mathbf{3 5 4}$ & $4-\mathrm{SO} 2 \mathrm{Me}$ & $4-\mathrm{F}$ & $\mathrm{Br}$ & 8.00 \\
\hline
\end{tabular}

